\title{
Stem Cells, A Reservoir for Life
}

\author{
Abdolamir Allameh ${ }^{1, *}$ \\ ${ }^{1}$ Department of Clinical Biochemistry, Faculty of Medical Sciences, Tarbiat Modares University, Tehran, IR Iran \\ ${ }^{*}$ Corresponding author: Abdolamir Allameh, Department of Clinical Biochemistry, Faculty of Medical Sciences, Tarbiat Modares University, Tehran, IR Iran. Tel: + 98-2188013030, Fax: \\ +98-2182883558, Email: allameha@modares.ac.ir
}

Received: July 20, 2013; Revised: July 22, 2013; Accepted: July 22, 2013

Keywords: Differentiation; Regenerative Medicine; Stem cell

The stem cells can be considered as the miracle of creation, because, they are a major reservoir of the bioactive factors, molecules and cells. These reservoir cells play a major role in development and growth of the tissues and organs. Regardless of the differences in differentiation capacity of embryonic and adult stem cells, both the cells are rich sources of stem cells with therapeutic application. Moreover, manipulation of adult stem cells and reprogramming or reverting embryonic-like state cells can be considered as additional tools for stem cell applications. Stem cells from different sources with different potential (plasticity) may be used to obtain ideal cell lineage with a defined function. Bone marrow, embryo and cord blood are the major stem cell sources which are believed to act systemically for regeneration of tissues and organs in an organism. In addition to embryonic and adult stem cells, there are other sources such as skin, which is a good source of adult stem cells involved in local repair and regeneration of epidermal and dermal tissues (1).

Embryonic stem cells (ES): ES cells are pluripotent stem cells derived from the inner cell mass of a blastocyst. The pluripotency, and their ability to replicate indefinitely are two distinctive properties of the ES.

ES cells are readily differentiated into primary germ layers, viz., ectoderm, endoderm, and mesoderm. Pluripotency distinguishes ES cells from adult stem cells which are multipotent cells with the capacity to produce limited number of cell types (2). The application of pluripotent stem cells in treatment of blood and immune-system related genetic disorders such as; juvenile diabetes; Parkinson's; blindness, spinal cord injuries and cancers is well established. The transplantation of oligodendrocytes derived from human ES cells into spinal cord-injured individuals was the world's first human ES cell human trial approved by FDA.

The diversity of the stem cells present in different tis- sues implies that the body reserves all the cell types with stemness properties to guarantee important physiological processes and regeneration of organs.

Placental stem cells: The placenta has become useful source of stem cells that offer advantages in terms of proliferation and plasticity when compared with adult cells (3). The epithelia, haematopoietic and mesenchymal stem cells are major stem cells isolated from the placenta. Adult stem cells: The pluripotency of adult stem cells isolated from different tissues was approved by showing their differentiation potential into cell types from different germ layers. For example, neural stem cells derived from ectoderm can differentiate into ectoderm, mesoderm, and endoderm.

Bone marrow: Bone marrow is a rich source of two important adult stem cell lineages: Hematopoietic Stem Cells (HSCs) and Mesenchymal Stem Cells (MSCs).

Hematopoietic stem cells (HSCs); HSCs can give rise to all the blood cell types. The HSCs with self-renewal capacity can differentiate into different types of blood cells, including those involved in the human immune system. There are three types of HSC transplants; syngeneic, autologous, and allogeneic transplants. Human bone marrow grafting is currently considered as significant progress in HSCs therapy. HSCs are also applied in reconstitution of damaged hematopoietic cells and restoring the immune system following chemotherapy in certain diseases.

Mesenchymal Stem Cells (MSCs): There are different sources of MSCs and their isolation is relatively easy. The placenta, adipose tissue (4), lung, bone marrow and blood, Wharton's jelly from the umbilical cord, dental pulp and periodontal ligament are often used to isolate MSCs. These cells can be differentiated into various cell lineages and considering their rapid proliferation, multipotency and immunomodulatory properties they are

Implication for health policy / practice / research / medical education:

The implication of this study is to know recent developments in the stem cell technology and applications. To briefly describe the importance of different types of stem cells with their differentiation potential in cell therapy and regeneration medicine.

Copyright (c) 2013, National Institute of Genetic Engineering and Biotechnology; Published by Kowsar Corp. This is an Open Access article distributed under the terms of the Creative Commons Attribution License (http: // creativecommons.org / licenses / by / 3.0), which permits unrestricted use, distribution, and reproduction in any medium, provided the original work is properly cited. 
widely used for treatment of various diseases. So far several cell types have been differentiated from MSCs.

Mammary stem cells: These cells provide the source of cells for growth of the mammary gland during puberty and gestation. These cells have been isolated from human and mouse tissue as well as from cell lines originated from the mammary gland that can give rise to both the luminal and myoepithelial cell types of the gland.

Neural stem cells: The presence of stem cells in the mature animal brain tissue indicates that during the neurogenesis process generation of new neurons continues into adulthood.

The subventricular zone, which lines the lateral ventricles, and the dentate gyrus of the hippocampal formation are two major regions of the brain comprising adult neurogenesis. The generation of new neurons in the hippocampus is well established but under certain conditions such as tissue injuries in ischemia, neurogenesis can be induced in other regions in the brain.

Testicular cells or spermatogonial progenitor cells: These multipotent stem cells have been derived from spermatogonial progenitor cells found in the testicles. Also multipotent stem cells have been derived from germ cells found in testicles which are known as Human Adult Germline Stem Cells (GSCs). The stem cells and the cells derived from them are capable of directly contribute to organ and tissue repair and regeneration. Intestinal stem cells, olfactory adult stem cells and hair follicles cells are other sources which have been used to isolate different stem cells. The advantage of these cells is that they can be harvested with ease from the patients. Perhaps, with the advancements made in the stem cell technologies, the scientists are becoming more optimistic about the therapeutic potential and safety of the stem cell therapy.

The existence of stem cells in different tissues, with proliferation and differentiation capacity indicates that activation of these cells is regulated via pathways linked to development, repair and regeneration. However despite the advancements made in stem cell differentiation, no studies have succeeded in generating a functional organ such as a human liver. The very recent breakthrough in generation of a three-dimensional vascularized and functional human liver from human pluripotent stem cells (iPSCs) by transplantation liver buds made in vitro-grown organ buds is promising for chronic liver diseases (5).

\section{Financial Disclosure}

The author declares no competing financial interests.

\section{References}

1. Chunmeng S, Tianmin C. Skin: a promising reservoir for adult stem cell populations. Med Hypotheses. 2004;62(5):683-8.

2. Embryonic stem cell. Available from: http: // en.wikipedia.org / wiki / Embryonic_stem_cell.

3. Pipino C, Shangaris P, Resca E, Zia S, Deprest J, Sebire NJ, et al. Placenta as a reservoir of stem cells: an underutilized resource? $\mathrm{Br}$ Med Bull. 2013;105:43-68.

4. Onate B, Vilahur G, Ferrer-Lorente R, Ybarra J, Diez-Caballero A, Ballesta-Lopez C, et al. The subcutaneous adipose tissue reservoir of functionally active stem cells is reduced in obese patients. FASEB J. 2012;26(10):4327-36.

5. Takebe T, Sekine K, Enomura M, Koike H, Kimura M, Ogaeri T, et al Vascularized and functional human liver from an iPSC-derived organ bud transplant. Nature. 2013;499:481- 484. 